\section{De nouvelles techniques pour dévoiler le rôle des cellules gliales du cerveau}

Anne-Laure Hemonnot-Girard ${ }^{1}$, Lucile Ben Haim ${ }^{2 *}$, Carole Escartin ${ }^{2 *}$, Hélène Hirbec ${ }^{1 *}$

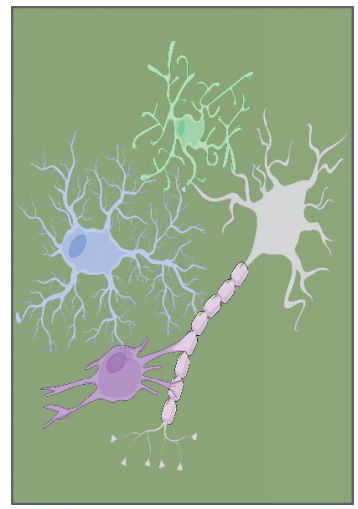

${ }^{I}$ IGF, Univ Montpellier, CNRS, Inserm, Montpellier, France. ${ }^{2}$ Université Paris-Saclay, CEA, CNRS, MIRCen (Molecular Imaging Research Center), Laboratoire des maladies neurodégénératives, 92265, Fontenayaux-Roses, France.

* Ces auteures ont contribué à part égale au manuscrit hirbec.helene@igf.cnrs.fr

aussi appelées cellules NG2 car elles expriment le protéoglycane NG2 (neuron-glial antigen 2). Les astrocytes ont un rôle majeur dans I'homéostasie du SNC; les oligodendrocytes assurent la myélinisation et apportent un support trophique aux axones; et les OPC peuvent générer de nouveaux oligodendrocytes tout au long de la vie.

Les microglies, quant à elles, ont une origine mésodermique et dérivent des précurseurs myéloïdes fœtaux du sac vitellin. Ces précurseurs envahissent le SNC au début de la période embryonnaire, se disséminent dans l'ensemble du cerveau et de la moelle épinière où ils acquièrent un phénotype microglial. Les microglies sont les principales cellules immunitaires du SNC, elles en assurent la surveillance et la défense.

Comme les neurones, les cellules gliales ont commencé à être étudiées au $x \mid x^{e}$ siècle en utilisant un simple microscope et des techniques de coloration. Contrairement aux neurones, ces cellules ne sont pas ou sont peu excitables. Elles étaient considérées, jusqu'au milieu du $\mathrm{xx}^{\mathrm{e}}$ siècle, comme assurant principalement des fonctions de support physique vis-à-vis des neurones (glie vient du grec $\gamma \lambda$ oró $\varsigma_{-g l o i o s-}-$, qui signifie colle). À partir des années 1950 , l'avènement de nouvelles techniques d'électrophysiologie et d'immunohistochimie a cependant permis à la gliobiologie de se développer pour devenir aujourd'hui un champ d'étude à part entière au sein des neurosciences.

Ces trente dernières années, les découvertes scientifiques dans ce domaine émergent se sont enchainées, démontrant que les cellules gliales ont des rôles importants dans le développement, la plasticité, le vieillissement et les maladies du SNC [2]. Ces cellules apparaissent comme hautement spécialisées et sont des partenaires actifs des 


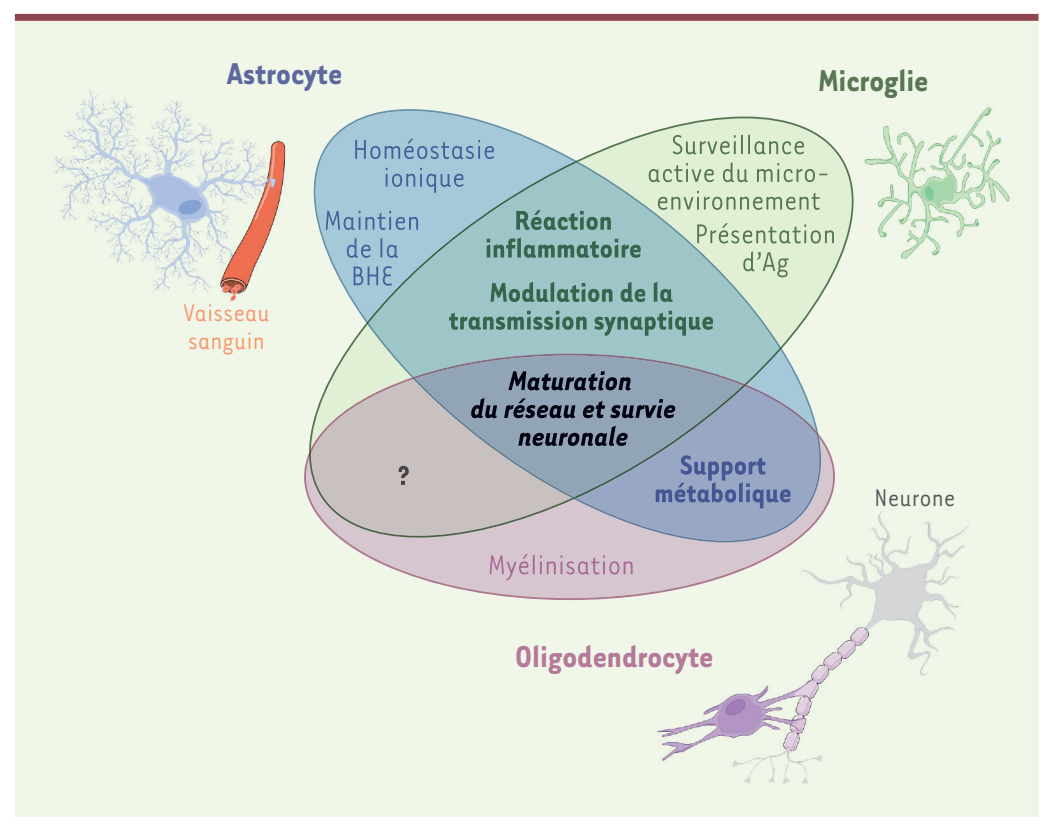

Figure 1. Principales fonctions des cellules gliales dans le SNC. Ce diagramme de Venn représente les principales fonctions physiologiques assurées par les différents types de cellules gliales dans le cerveau. Certaines de ces fonctions (en gras sur la figure) sont partagées par plusieurs types cellulaires. Bien qu'ils aient des fonctions spécifiques, tous les types de cellules gliales concourent à la survie neuronale et à la maturation des réseaux neuronaux. BHE : barrière hématoencéphalique; $\mathrm{Ag}$ : antigène.

\section{Les méthodes de culture en 《 2D 》}

L'intérêt majeur des cultures en deux dimensions (2D) est de permettre des analyses à moyen et haut débit pour tester l'efficacité d'agents thérapeutiques. Ces approches permettent d'obtenir des preuves de concept et de valider des médicaments candidats avant d'utiliser des

neurones. Les différents types de cellules gliales ne constituent cependant pas des populations homogènes, mais sont composées de différents sous-types qui varient selon le stade de développement, la région cérébrale ou encore du sexe. Cette hétérogénéité permet à ces cellules une adaptation fonctionnelle à leur microenvironnement. $\varepsilon n$ dehors de leurs rôles physiologiques, elles peuvent réagir à de nombreux stimulus pathologiques et contribuent aux processus neuroinflammatoires qui sont communs à de nombreuses pathologies du SNC. La découverte de nouvelles fonctions remplies par les cellules gliales ouvre des perspectives inédites permettant de mieux comprendre le fonctionnement et le dysfonctionnement du SNC. Pour autant, les cellules gliales n'ont certainement pas dévoilé tous leurs secrets.

Dans cette synthèse inspirée d'une revue plus exhaustive sur le sujet [3], nous décrivons les avancées techniques les plus remarquables, qui ont permis aux neuroscientifiques d'en apprendre plus sur les origines et les rôles fonctionnels des cellules gliales. Pour chacune des méthodes décrites, nous présenterons une sélection d'études qui illustrent l'intérêt de la technique et des revues détaillées pour approfondir le sujet. Nous mentionnerons également les avantages, les limites de ces approches et les développements futurs envisagés pour chacune. Dans un avenir proche, ces techniques émergentes permettront de révéler encore davantage l'importance des cellules gliales dans les (dys)fonctions cérébrales.

\section{La glie... c'est dans la boite! Les nouveaux modèles in vitro}

Les cellules gliales ont d'abord été mises en culture in vitro pour fournir un support trophique aux cultures de neurones. La mise en évidence d'un rôle fonctionnel crucial pour ces cellules a néanmoins motivé les chercheurs à obtenir des cultures pures pour pouvoir les étudier spécifiquement. La compréhension des mécanismes du développement du SNC et de la réaction gliale a ensuite conduit à des avancées majeures dans les méthodes de culture de ces cellules. systèmes in vitro plus intégrés, reproduisant mieux la complexité du SNC, ou des modèles in vivo.

L'immunopanning est une technique efficace pour obtenir des cellules purifiées. Elle repose sur la reconnaissance par des anticorps, adsorbés sur des boites de Pétri, d'antigènes spécifiques de chaque type cellulaire [4]. Cette technique permet d'obtenir des quantités de cellules purifiées compatibles pour des expériences à moyen débit. L'immunopanning a ensuite été adapté afin de réaliser une séparation « magnétique » des cellules, fondée sur l'utilisation d'anticorps couplés à des micro-billes magnétiques, une méthode qui s'avère plus efficace en termes de rendement.

Les milieux de culture ont eux aussi dû être adaptés pour la culture des cellules gliales. L'utilisation de facteurs de croissance bien définis, la réduction des taux de glucose ou d'albumine, ainsi que l'absence de sérum [5], permettent aujourd'hui de cultiver chaque type cellulaire dans un état aussi physiologique que possible, un prérequis important pour étudier les propriétés des cellules gliales.

\section{Cultures de cellules humaines}

Les méthodes de culture développées pour les cellules primaires de rongeur ont été appliquées avec succès aux cellules fœtales et aux cellules nerveuses humaines adultes. Cependant, pour des raisons légales, éthiques et logistiques, l'utilisation de ces cellules est très limitée. Les techniques de reprogrammation cellulaire ont été adaptées aux cellules humaines. Elles permettent de générer des cellules souches pluripotentes (hiPSC, pour human induced pluripotent stem cell) à partir de fibroblastes de peau ou de cellules sanguines. Ces cellules peuvent ensuite être différenciées en oligodendro- 


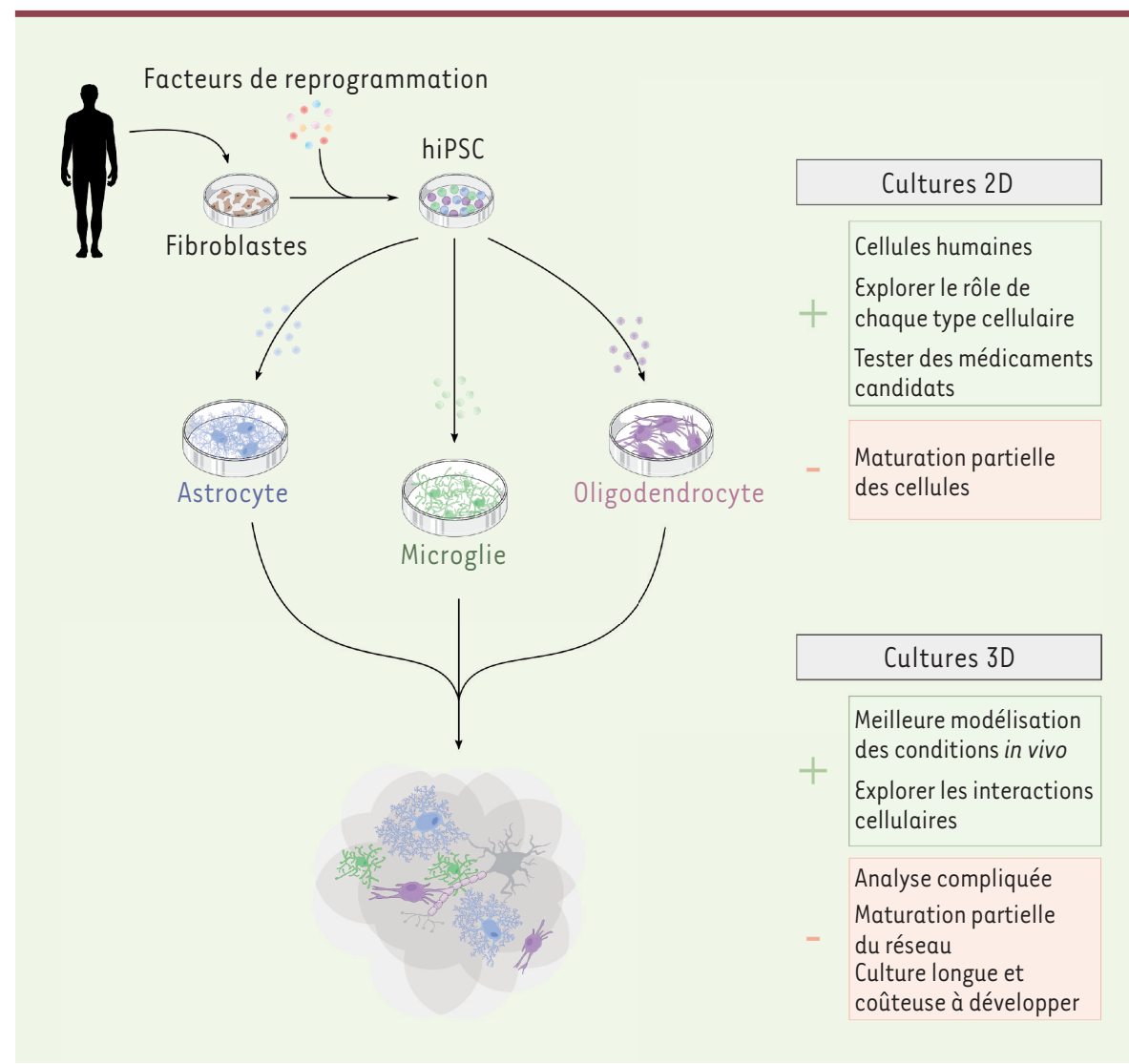

Figure 2. Les cellules pluripotentes induites humaines, un outil translationnel pour étudier les cellules gliales in vitro et ex vivo. L'application d'un cocktail de facteurs sur des cellules somatiques provenant de donneurs permet de les reprogrammer et d'obtenir des cellules souches pluripotentes (hiPSC). Ces hiPSC peuvent ensuite, grâce à des facteurs spécifiques, être différenciées dans les différents types de cellules gliales. L'ensemble de ces cellules humaines peuvent ensuite être assemblées sous forme de culture en trois dimensions (3D). Chacun de ces types de culture présentent des avantages et des inconvénients. Le modèle doit donc être sélectionné en fonction de la question biologique posée et en prenant en considération les caractéristiques intrinsèques de chaque mode de culture.

cytes [6], en astrocytes [7] et depuis peu, en microglies, grâce à des protocoles inspirés des processus de différenciation survenant in vivo [8] (Figure 2). Les cellules gliales dérivées des hiPSC offrent des systèmes puissants pour comprendre les cellules gliales humaines et leurs rôles dans le développement et les maladies du SNC. Cependant, ces cellules dérivées d'hiPSC restent très éloignées des cellules humaines telles qu'on les observe in vivo, que ce soit au niveau morphologique, moléculaire ou fonctionnel. Ces cultures sont coûteuses, longues à mettre en place et sont surtout réservées aux études translationnelles dont le but est de développer de nouvelles thérapies ou d'étudier des pathogènes spécifiques à l'homme.

De nouvelles méthodes de culture émergent, essayant de recréer les interactions complexes entre les divers types cellulaires du SNC. Les cultures 2D peuvent être combinées et organisées en cultures multicellulaires intégrées. Certaines de ces cultures en trois dimensions (3D) s'organisent de manière autonome, formant ainsi des organoïdes $(\rightarrow)$.

$\rightarrow$ Voir la série Organoïdes, $\mathrm{m} / \mathrm{s} \mathrm{n}^{\circ} 5$ (mai 2018) à $\mathrm{m} / \mathrm{s}$ $n^{\circ} 10$ (octobre 2020)
D'autres systèmes utilisent des matrices extracellulaires synthétiques [9]. Il est ainsi désormais possible d'obtenir des cultures complexes intégrant des neurones, des astrocytes, des oligodendrocytes et des cellules microgliales [10]. Dans cet environnement tridimensionnel, les cellules gliales arrivent à maturation plus rapidement et acquièrent des propriétés moléculaires et fonctionnelles plus proches des cellules in vivo, même si leur niveau de développement est loin d'être complet. Ce type de culture constitue un outil sans précédent pour l'étude du développement cérébral, des pathologies qui y sont liées, mais peut aussi permettre d'étudier les phases précoces de maladies neurodégénératives, comme la maladie d'Alzheimer ou de Parkinson [10].

\section{Nouveaux outils pour observer et manipuler les cellules gliales}

La plupart des études menées sur la glie ont jusqu'à présent utilisé des souris transgéniques permettant de visualiser (après transduction d'un gène codant un rapporteur fluorescent) ou de manipuler (par le système de recombinaison CreLox ${ }^{2}$ ) les cellules gliales. Aujourd'hui, la combinaison de ces approches transgéniques avec de nouveaux outils moléculaires permet de disséquer plus précisément le rôle des cellules gliales (Figure 3 ).

\section{Vecteurs viraux}

Les vecteurs viraux sont une option intéressante pour moduler l'expression des gènes gliaux in vitro et in vivo [11]. Ce sont des outils polyvalents, faciles à produire et utilisables en clinique, car fondés sur des virus non réplicatifs [12]. Deux types de vecteurs viraux sont principalement utilisés pour cibler les cellules du SNC : les vecteurs lentiviraux (LV) et les vecteurs viraux adéno-associés recombinants (rAAV). Chaque

${ }^{2}$ Le système Cre-Lox est une technique de recombinaison permettant d'effectuer 


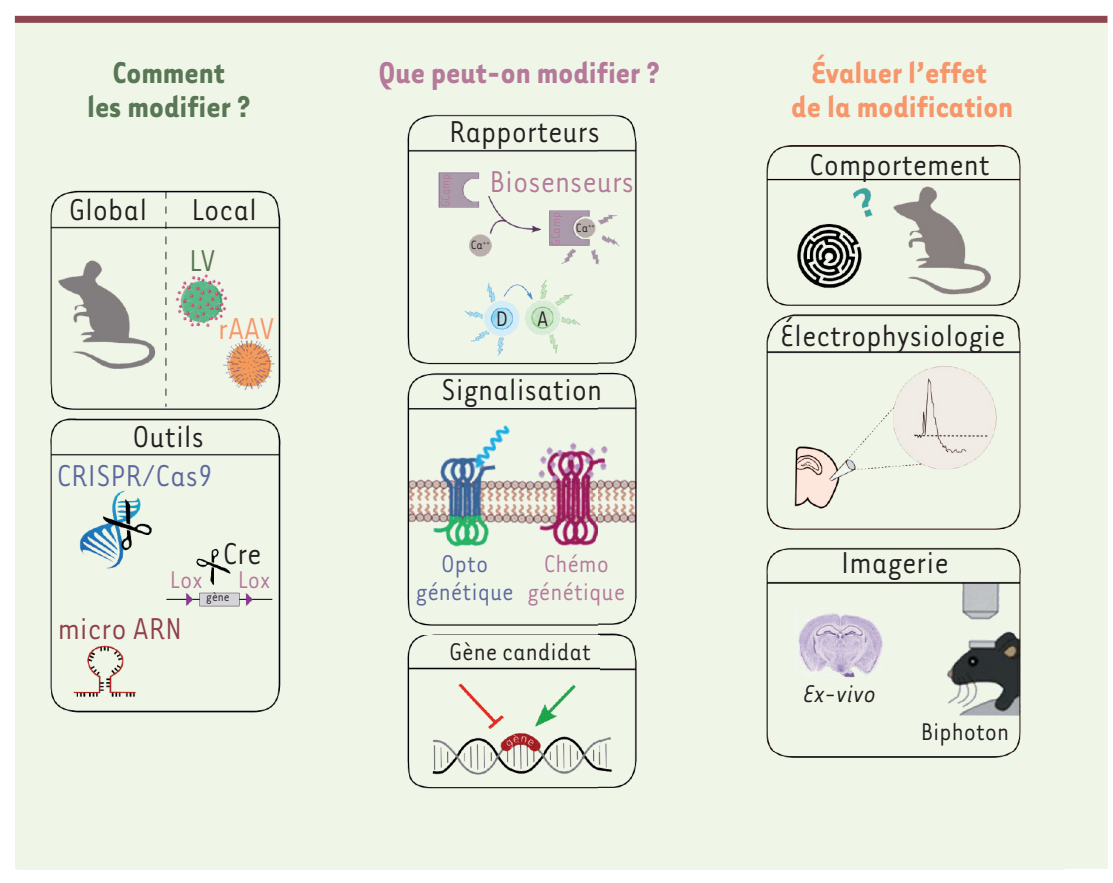

Figure 3. Modifier les cellules gliales pour mieux les étudier. Les cellules gliales peuvent être modifiées en utilisant des stratégies de ciblage globales (souris transgéniques) ou locales (injection de vecteurs lentiviraux [LV] ou d'adéno-viraux associés recombinants [rAAV]). Différents systèmes permettent de modifier l'expression génique dans les cellules gliales (système CRISPR/Cas9, Cre-Lox, microARN). Ces outils peuvent être utilisés pour la visualisation des cellules gliales (gènes rapporteurs, biosenseurs), leur modulation fonctionnelle (outils opto- et chémogénétiques) ou moléculaire (ciblage de gènes candidats). Enfin, l'effet de ces manipulations peut être étudiée à l'échelle de l'animal (comportement), des réseaux neuronaux (électrophysiologie) et de la cellule (imagerie, analyse post-mortem).

système a des spécificités propres en termes de capacité de clonage, d'intégration au génome, de tropisme, de diffusion et d'induction de réponse immunitaire. L'utilisation de promoteurs spécifiques ou de stratégies de dé-ciblage permet en outre d'augmenter la spécificité cellulaire de ces vecteurs. Si des outils viraux sont disponibles pour cibler efficacement les astrocytes et les oligodendrocytes, les cellules microgliales et les $O P C$ restent plus difficiles à transduire.

Les vecteurs viraux ciblant les cellules gliales ont de nombreuses applications, comme l'expression de rapporteurs fluorescents, de biosenseurs ou de constructions opto- et chémogénétiques (Figure 3). Les vecteurs viraux sont aussi utilisés pour manipuler l'expression de gènes d'intérêt, inactiver ou remplacer des gènes cibles, à l'aide de microARN ou du système CRISPR/Cas9 $[46]^{3}(\rightarrow)$.

\section{$(\rightarrow)$ Voir le Repères de B.Jordan, page 77 de ce numéro}

\section{Imagerie cellulaire}

Le développement concomitant d'indicateurs fluorescents, codés génétiquement, et de nouvelles techniques d'imagerie optique a considérablement accéléré l'étude des cellules gliales. Cela a notamment permis de révéler la façon dont elles interagissent avec les neurones, en conditions physiologiques et pathologiques.

Différents types d'indicateurs permettent de visualiser ou de mesurer des évènements de signalisation cellulaire, par la détection de messagers intracellulaires ( $\mathrm{AMPc}, \mathrm{Ca}^{2+}$ ) ou de transmetteurs extracellulaires (glutamate, acide $\gamma$-aminobutyrique [GABA] ou ATP), avec une résolution spatiale et temporelle élevée [13]. Ces indicateurs peuvent être exprimés grâce à des approches génétiques ou de transductions

${ }^{3}$ Le système d'édition génomique CRISPR/Cas9 permet d'effectuer des modifications génétiques ciblées grâce à des ciseaux moléculaires spécifiques. Cette découverte majeure, réalisée par $\varepsilon$. Charpentier et J. Doudna a été récompensée par le Prix Nobel de Chimie en octobre 2020 [46]. virales, et utilisés pour des études in vitro (sur des cellules en culture), ex vivo (sur des tranches de cerveaux) et in vivo (y compris sur des animaux vigiles ${ }^{4}$ ) dans des types cellulaires spécifiques. Ces indicateurs ont notamment permis de révéler l'importance de la signalisation calcique astrocytaire dans la communication entre neurones et astrocytes [13].

En plus d'indicateurs fonctionnels, le développement de techniques d'imagerie cellulaire à haute résolution permet de visualiser les cellules gliales et de mesurer des comportements cellulaires complexes, comme la surveillance du parenchyme cérébral et la phagocytose par la microglie [14], ou la dynamique de myélinisation des axones par les oligodendrocytes [15].

\section{Outils opto- et chémogénétiques}

Les outils opto- et chémogénétiques sont utilisés pour activer ou inhiber les cellules gliales dans lesquelles ils sont exprimés, par des approches transgéniques ou virales. Les outils optogénétiques sont des récepteurs sensibles à la lumière. Ils conduisent à l'activation ou à l'inhibition des cellules par modulation de courants ioniques membranaires ou de voies de signalisation intracellulaires [16]. La source de lumière est généralement délivrée via l'objectif du microscope ou via l'implantation d'une fibre optique dans le parenchyme cérébral [17]. Cette approche a permis de montrer que les astrocytes régulent des processus physiologiques complexes, comme la respiration [18], le sommeil [19] ou encore la prise alimentaire [20].

\footnotetext{
4 Vivants et en éveil.
} 
Les outils chémogénétiques sont, quant à eux, des récepteurs pouvant être couplés à différentes protéines $G$. Ils permettent l'activation de voies de signalisation intracellulaires après l'administration d'un ligand exogène, le clozapine-N-oxide (CNO) [21]. Le CNO peut être administré directement dans le cerveau, par injection par voie générale ou dans l'eau de boisson. Contrairement aux outils optogénétiques qui permettent des stimulations à l'échelle de la milliseconde, la modulation chémogénétique est de longue durée et se prolonge entre 30 min et $2 \mathrm{~h}$ après l'administration du CNO, ce qui est compatible avec la réalisation de tests comportementaux [17]. Ces outils récents ont permis de montrer que les astrocytes peuvent moduler la mémoire [22], ainsi que le comportement de peur [23] ou d'addiction [24] dans différentes régions du cerveau.

Ces approches émergentes ont cependant des limites à prendre en compte. Les vecteurs viraux peuvent en effet déclencher des réactions inflammatoires ; ils ont une capacité de clonage limitée (en particulier pour les rAAV); et ils nécessitent la réalisation de chirurgie pour les injecter directement dans le cerveau. De nouveaux vecteurs permettant le passage de la barrière hémato-encéphalique ont récemment été développés : après injection intraveineuse, ils rendent possible la transduction de cellules gliales dans des zones étendues du SNC [22]. Les outils opto- et chémogénétiques sont, jusqu'à présent, très majoritairement utilisés pour étudier les astrocytes, même s'ils commencent à être appliqués à d'autres types de cellules gliales. Contrairement aux neurones, les changements ioniques induits par la stimulation optogénétique ne reflètent probablement pas la signalisation caractéristique des cellules gliales. L'utilisation de ces outils peut également avoir des effets indésirables non spécifiques (acidification, augmentation de température dans le tissu, inflammation due à l'injection systémique de CNO, etc.) [13]. Dans le futur, le développement de nouveaux biosenseurs plus physiologiques et plus spécifiques, pour tous les types de cellules gliales, permettra de mieux comprendre le fonctionnement de ces cellules.

\section{Établir la carte d'identité moléculaire des cellules gliales grâce aux approches à haut débit}

\section{Profilage du transcriptome des cellules gliales}

Les approches de séquençage à haut-contenu permettent, à partir des ARN messagers (ARNm) extraits, de déterminer de manière quasi exhaustive le transcriptome ${ }^{5}$ d'un tissu, d'un groupe de cellules ou même d'une cellule unique (Figure 4). Le développement de ces technologies et la diminution de leur coût a permis, dans les dix dernières années, d'établir le profil des gènes exprimés dans de nombreux types cellulaires et selon différentes conditions.

L'équipe de Ben Barres (Département de neurobiologie, Stanford University School of Medicine, Stanford, Californie, États-Unis) (décédé en 2017) a été pionnière dans la caractérisation du transcriptome des astrocytes, des microglies, des oligodendrocytes et des OPC [25]. Ces études ont permis d'identifier de nouveaux marqueurs plus spécifiques

${ }^{5}$ Le transcriptome d'une cellule ou d'une population de cellules représente le répertoire des gènes exprimés par ces cellules à un temps donné. de chacun des types de cellules gliales, facilitant leur étude ultérieure. En caractérisant, pour chaque type de cellules gliales, des profils spécifiques selon le stade de développement, les régions cérébrales ou encore le sexe, ces approches ont aussi permis de révéler que ces cellules étaient très intimement adaptées à leur environnement local.

Ces techniques sont aussi utiles pour étudier les modifications transcriptionnelles des cellules gliales dans diverses conditions pathologiques. Elles ont permis de montrer qu'elles ne réagissent pas selon une loi «du tout ou rien », mais adoptent un état réactif qui dépend de la nature du stimulus pathologique, de son intensité, de sa durée, de la région cérébrale touchée, etc. Cette diversité de la palette réactionnelle gliale représente à la fois un défi et une chance pour la mise au point de nouvelles stratégies thérapeutiques qui, par exemple, cibleraient seulement certains états délétères. Les études des transcrits gliaux ont néanmoins permis d'identifier quelques caractéristiques communes de la réaction des cellules gliales, telles que la perte de certaines fonctions homéostatiques et l'acquisition de fonctions inflammatoires.

Grâce aux tous derniers développements techniques, il est maintenant possible d'établir le transcriptome d'une cellule unique (ou single-cell RNA-seq). Cette technologie est un outil puissant qui permet de décrypter l'hétérogénéité intrinsèque d'une population cellulaire et d'étudier précisément la diversité des réponses cellulaires au niveau individuel [26]. Cette méthode a récemment mis en évidence l'existence et l'évolution de plusieurs sous-types de microglies dans un modèle murin de la maladie d'Alzheimer [27]. Ces approches de séquençage à haut-débit peuvent aussi révéler des modifications épigénétiques, qui représentent des mécanismes importants pour la plasticité du SNC.

\section{Profilage du protéome des cellules gliales}

Le niveau d'expression des gènes peut être utilisé pour prédire l'état fonctionnel d'une population ou souspopulation cellulaire donnée. Néanmoins, les ARNm ne sont pas les effecteurs ultimes de la cellule et il peut exister d'importantes différences entre l'expression des gènes et celle des protéines qu'ils codent. Les protéines peuvent également subir des modifications post-traductionnelles qui affectent considérablement leur activité. Déterminer le répertoire de protéines et/ ou leurs modifications post-traductionnelles est bien plus complexe, puisque les protéines, contrairement aux acides nucléiques, ne peuvent pas être amplifiées.

Pour étudier à grande échelle les protéines exprimées par une population cellulaire spécifique, deux 


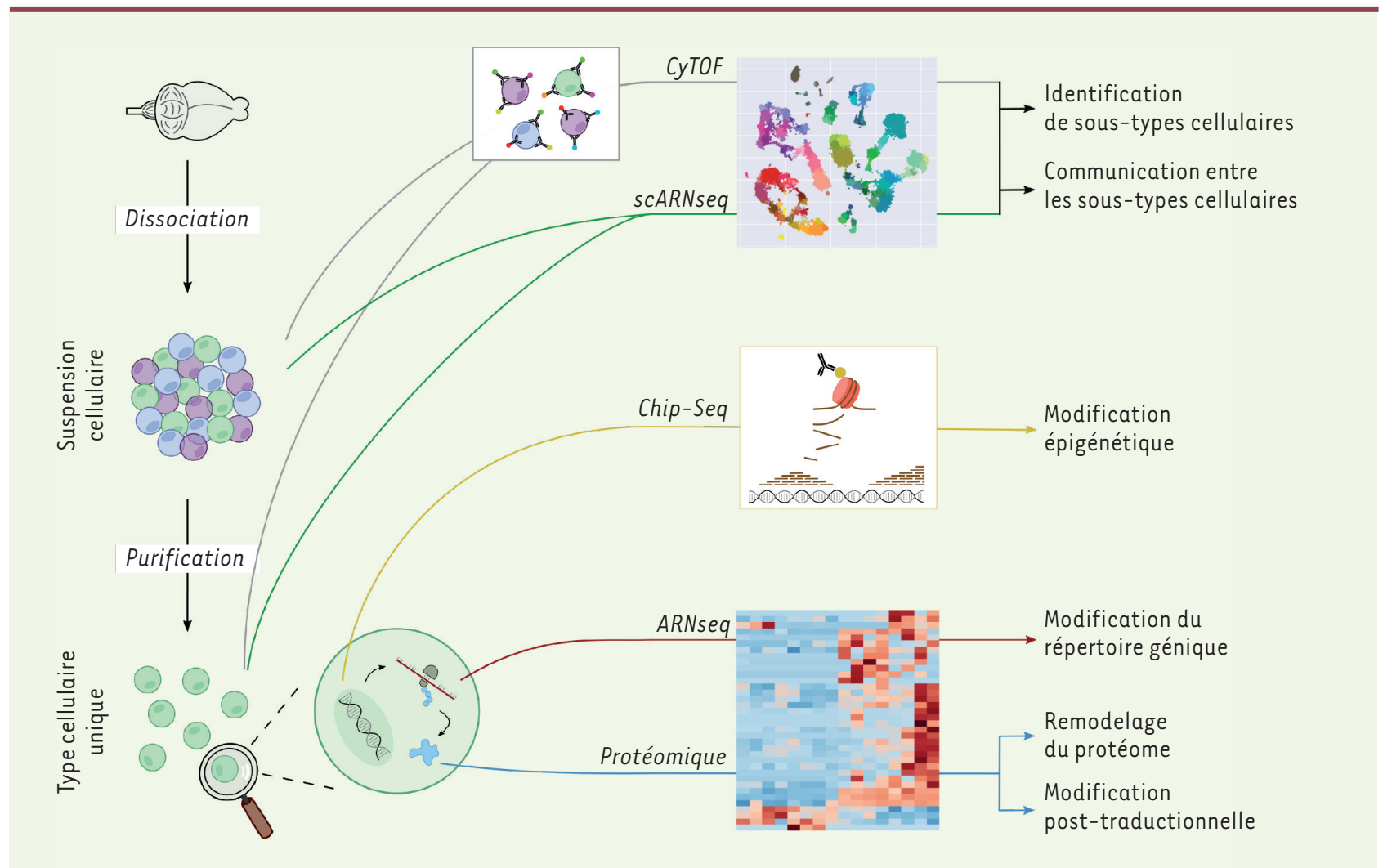

Figure 4. Approches moléculaires à haut débit pour mieux caractériser les cellules gliales. Les approches à haut-débit ont pour objectif de caractériser des populations de cellules ou des types cellulaires en n'émettant pas ou peu d'hypothèses a priori. Pour ces approches, les cellules doivent être mises en suspension et éventuellement purifiées. Ces approches sont fondées sur la quantification des niveaux d'expression des ARNm (RNAseq ou scRNAseq), des protéines (CyTOF ou protéomique), ou des marqueurs épigénétiques (Chip-Seq). Ces méthodes fournissent un grand nombre de données dont l'analyse nécessite l'utilisation d'approches bioinformatiques et statistiques sophistiquées.

approches sont possibles: la spectroscopie de masse et la cytométrie de masse (ou CyTOF pour cytometry by time of flight). La première méthode permet l'exploration combinée de centaines ou de milliers de protéines, les derniers développements techniques ayant permis de gagner en sensibilité et en précision [28]. Par cette approche, Sharma et al. [29] ont, en analysant des astrocytes, des oligodendrocytes et des microglies isolés de plus de 10 régions cérébrales, établi le premier véritable catalogue protéomique des cellules du cerveau. Ce type d'analyse a aussi permis d'identifier des modifications microgliales précoces dans un modèle murin de la maladie d'Alzheimer [30].

Comme en cytométrie classique, la CyTOF est fondée sur la reconnaissance d'épitopes connus par des anticorps spécifiques. Cependant, à la différence de la cytométrie en flux qui utilise des anticorps couplés à des fluorochromes, les anticorps utilisés avec la CyTOF sont couplés à des métaux rares, ce qui permet d'augmenter considérablement le nombre d'anticorps compatibles entre eux et utilisables simultanément, et autorise une analyse à grande échelle avec une résolution cellulaire, voire subcellulaire [31]. Cette approche a récemment établi que les microglies humaines sont phénotypiquement distinctes des macrophages périphériques [32].
Qu'elles ciblent les transcrits ou les protéines, les approches à haut débit permettent de générer un grand nombre d'informations. Ces méthodes ne sont toutefois pas dénuées d'inconvénients. Elles nécessitent des outils d'analyse performants et, idéalement, utilisables par des chercheurs qui ne sont pas bioinformaticiens. L'un des principaux problèmes de ces approches réside également dans le fait qu'elles nécessitent de dissocier les tissus pour obtenir des suspensions cellulaires purifiables. Ces procédures de dissociation peuvent induire une réaction des cellules et modifier leur état qui ne correspond donc que partiellement à leur état in vivo. Plus que celui des ARN cytosoliques, le séquençage des ARN nucléaires isolés à partir de noyaux de tissus rapidement congelés pourrait permettre de résoudre partiellement ce problème, mais seule une fraction des ARN se trouve dans le noyau. Par ailleurs, en dissociant les cellules, l'information liée à leur microenvironnement cérébral est perdue. Pour éviter ces écueils, les dernières innovations visent à développer la spatiotranscriptomique [33] et l'imagerie moléculaire par 


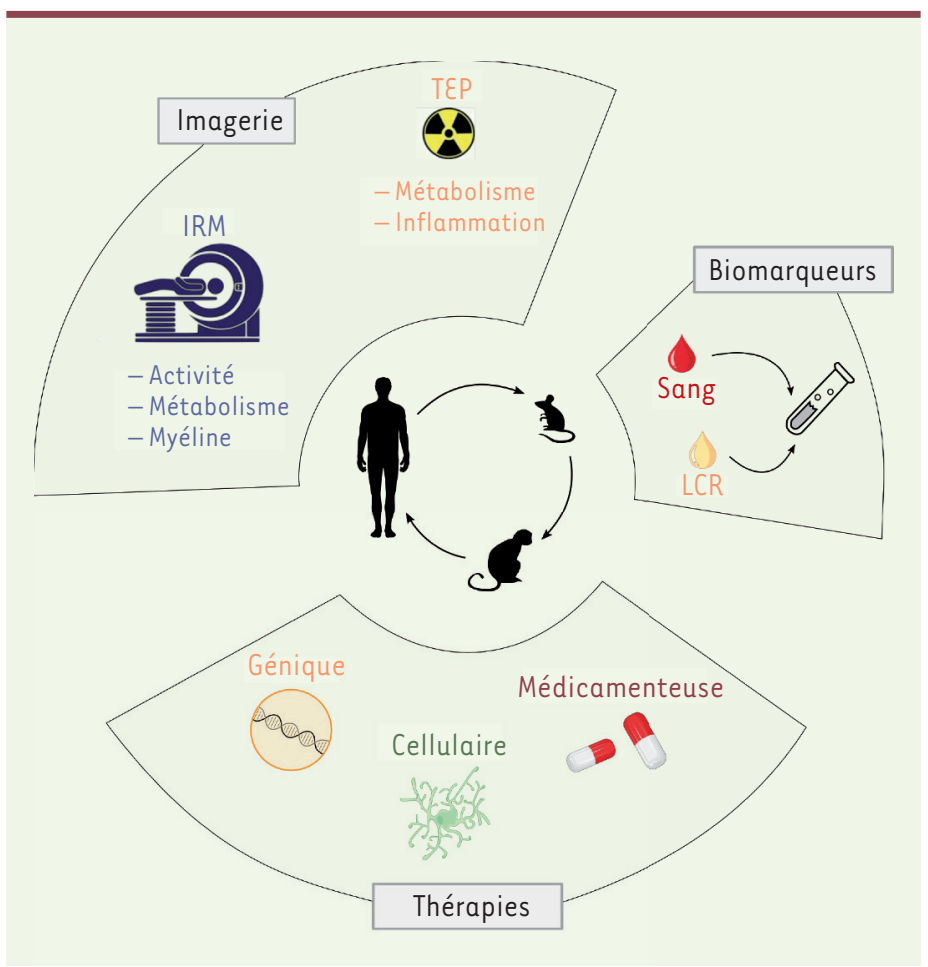

Figure 5. Approches translationnelles pour améliorer le transfert entre le laboratoire et la clinique. De nombreuses découvertes faites sur les cellules gliales en préclinique ont permis de développer des nouvelles méthodes d'imagerie cérébrale pour visualiser ou analyser la myéline, le métabolisme cellulaire, l'activité cérébrale, ainsi que de valider des bio-marqueurs sanguins ou dans le liquide céphalo-rachidien (LCR) et de proposer de nouvelles stratégies thérapeutiques.

spectrométrie de masse [34], deux approches qui permettent de réaliser ces analyses à haut-débit à partir de coupes histologiques et donc de conserver l'information spatiale. Cependant, ces approches sophistiquées et coûteuses ne sont pas encore très répandues dans les laboratoires.

\section{Les cellules gliales au chevet des patients}

Les cellules gliales occupent une importance grandissante en clinique, du fait du développement de biomarqueurs gliaux et de l'imagerie cérébrale non invasive fondée sur les cellules gliales, mais aussi parce que leur ciblage à des fins thérapeutiques est désormais possible [35] (Figure 5).

\section{L'imagerie cérébrale est parfois fondée sur les cellules gliales}

Plusieurs techniques d'imagerie cérébrale développées en recherche pré-clinique et utilisées en clinique sont fondées sur la détection - directe ou indirecte - des cellules gliales. Par exemple, l'imagerie par résonance magnétique (IRM) de la myéline permet de suivre les lésions et leur remyélinisation [36]; la tomographie par émission de positons (TEP) de la «neuroinflammation » vise à localiser et mesurer la réactivité des cellules microgliales et des astrocytes [37]. À noter que la mesure de l'activité cérébrale, par quantification de la consommation de $\left[{ }^{18} \mathrm{~F}\right]$-désoxyglucose en TEP ou par la détection de la dé-oxyhémoglobine par IRM fonctionnelle, sont, en fait, fortement régulées par les cellules non neuronales péri-vasculaires (dont les astrocytes) qui contrôlent le flux sanguin cérébral et l'entrée de glucose dans le cerveau [38].

Des efforts importants sont maintenant menés pour développer des méthodes: 1) plus sélectives d'un type de cellule gliale (par exemple, microglie versus astrocyte réactif, qui ont des rôles différents mais coexpriment certains marqueurs détectés en TEP) ; 2) plus spécifiques d'un état (par exemple, microglie réactive versus homéostatique ou myéline néoformée versus myéline intacte); ou 3) ayant une valeur pronostique ou diagnostique (présence d'un sous-type de cellules gliales associé à une progression favorable d'une maladie).

\section{Les cellules gliales comme cibles thérapeutiques}

De nombreuses études révèlent le rôle crucial de différents types de cellules gliales dans les pathologies du cerveau. Différentes stratégies thérapeutiques sont déployées pour cibler ces cellules par des approches médicamenteuses ou par transfert de gènes, afin de favoriser leurs fonctions homéostatiques ou trophiques vis-à-vis des neurones [39]. Aux progéniteurs neuronaux qui sont greffés dans les cerveaux lésés ou malades, sont maintenant associés des progéniteurs gliaux pour qu'ils se différencient en astrocytes et favorisent la survie et le bon fonctionnement des neurones greffés. Deux récentes études ont notamment montré que la greffe d'astrocytes reprogrammés permettait de remplacer les neurones ayant dégénérés dans des modèles murins de la maladie de Parkinson et ainsi de de corriger les symptômes moteurs $[40,41]$. Ces stratégies innovantes sont en cours de validation pré-clinique ou commencent à faire l'objet d'essais cliniques. L'avenir proche verra probablement le développement de stratégies glio-centrées pour les maladies du cerveau. De récentes études s'intéressent également au ciblage des cellules gliales pour le traitement de maladies neuropsychiatriques traditionnellement considérées comme purement «neuronales », telles que l'autisme, la schizophrénie ou la dépression [42].

\section{Neurosciences : le $\mathrm{xxl}^{\mathrm{e}}$ siècle sera glial}

Beaucoup de développements méthodologiques récents ont pu être appliqués à l'étude des cellules gliales, même si certains, comme les approches chémogénétiques, sont plus adaptés à un sous-type donné. Les cellules gliales n'ont pas fini de nous étonner, et 
certaines, moins étudiées, comme les tanycytes (SNC) ou les cellules gliales du système nerveux entérique (SNP), qui sont respectivement importantes pour la régulation de la prise alimentaire ou les réponses aux infections, commencent à peine à révéler leurs secrets.

Les cellules gliales humaines présentent des spécificités propres, nécessitant que les données pré-cliniques obtenues en utilisant des modèles animaux soient validées chez l'homme. L'utilisation d'échantillons (biopsies, liquide céphalorachidien, tissus post-mortem) de patients au profil clinique bien caractérisé se développe. En parallèle, de plus en plus de laboratoires intègrent l'analyse des cellules dérivées d'iPSC de patients.

La communauté de chercheurs travaillant sur les cellules gliales s'agrandit et tisse des liens de plus en plus étroits avec des disciplines aussi variées que la chimie, la physique, l'informatique et la statistique. De telles approches multidisciplinaires permettront de développer des outils encore plus performants et d'intégrer les énormes masses de données pour percer les mystères des cellules gliales, dont on ne soupçonnait pas l'importance il y a encore un demi-siècle $[43]^{6}(\rightarrow)$. $\diamond$

\section{$\rightarrow$ Voir l’éditorial de $Y$. Agid et X. Fan, $m / s n^{\circ} 3$, mars 2019 , page 199}

\section{SUMMARY}

New technologies to unveil the role of brain glial cells

Brain function relies on complex interactions between neurons and different types of glial cells, such as astrocytes, microglia and oligodendrocytes. The relatively young field of "gliobiology" is thriving. Thanks to various technical innovations, it is now possible to address challenging biological questions on glial cells and unravel their multiple roles in brain function and dysfunction.

We present several cutting-edge experimental approaches that are being used to understand the importance of glial cells in the central nervous system. We also illustrate how these techniques and findings on glial cells can then be translated to the clinics, to provide new biomarkers, non-invasive brain imaging approaches and hopefully in the near future, alternative therapeutic strategies. $\diamond$

\section{LIENS D'INTÉRÊT}

Les auteures déclarent n'avoir aucun lien d'intérêt concernant les données publiées dans cet article.

\section{RÉFÉRENCES}

1. Butt A, Verkhratsky A. Neuroglia: realising their true potential. Brain Neurosci Adv 2018 ; 2 : 2398212818817495.

2. Allen NJ, Lyons DA. Glia as architects of central nervous system formation and function. Science $2018 ; 362: 181-5$

3. Hirbec H, Deglon N, Foo LC, et al. Emerging technologies to study glial cells. Glia $2020 ; 68$ : $1692-$ 728.

4. Barres BA, Hart IK, Coles HS, et al. Cell death and control of cell survival in the oligodendrocyte lineage. Cell $1992 ; 70: 31-46$.

5. Bohlen CJ, Bennett FC, Tucker AF, et al. Diverse requirements for microglial survival, specification, and function revealed by defined-medium cultures. Neuron $2017 ; 94: 759-73$ e8.

6. Hu BY, Du ZW, Zhang SC. Differentiation of human oligodendrocytes from pluripotent stem cells. Nat Protoc $2009 ; 4: 1614-22$.

${ }_{6}$ Pour aller plus loin, nous recommandons la lecture des ouvrages suivants « L'homme glial » de Y. Agid et P. Magistretti [44] et «La myéline : le turbo du cerveau » de B. Zalc et F. Rosier [45].
7. Krencik R, Zhang SC. Directed differentiation of functional astroglial subtypes from human pluripotent stem cells. Nat Protoc $2011 ; 6: 1710-7$.

8. Muffat J, Li Y, Yuan B, et al. Efficient derivation of microglia-like cells from human pluripotent stem cells. Nat Med 2016 ; 22 : 1358-67.

9. Lancaster MA, Renner M, Martin CA, et al. Cerebral organoids model human brain development and microcephaly. Nature 2013 ; 501 : 373-9.

10. Korhonen P, Malm T, White AR. 3D human brain cell models: new frontiers in disease understanding and drug discovery for neurodegenerative diseases. Neurochem Int 2018; $120: 191-9$.

11. Blessing D, Deglon N. Adeno-associated virus and lentivirus vectors: a refined toolkit for the central nervous system. Curr Opin Virol 2016;21 : 61-6.

12. Palfi S, Gurruchaga JM, Lepetit H, et al. Long-term follow-up of a phase i/ ii study of prosavin, a lentiviral vector gene therapy for parkinson's disease. Hum Gene Ther Clin Dev 2018 ; 29 : 148-55.

13. Yu X, Nagai J, Khakh BS. Improved tools to study astrocytes. Nat Rev Neurosci $2020 ; 21: 121-38$.

14. Fuger $P$, Hefendehl JK, Veeraraghavalu $K$, et al. Microglia turnover with aging and in an Alzheimer's model via long-term in vivo single-cell imaging. Nat Neurosci 2017 ; 20 : 1371-6.

15. Hill RA, Li AM, Grutzendler J. Lifelong cortical myelin plasticity and agerelated degeneration in the live mammalian brain. Nat Neurosci $2018 ; 21$ : 683-95.

16. Fenno L, Yizhar 0, Deisseroth K. The development and application of optogenetics. Annu Rev Neurosci 2011 ; 34 : 389-412.

17. Goshen I. The optogenetic revolution in memory research. Trends Neurosci $2014 ; 37: 511-22$.

18. Gourine AV, Kasymov V, Marina N, et al. Astrocytes control breathing through pH-dependent release of ATP. Science 2010 ; 329 : 571-5.

19. Poskanzer KE, Yuste R. Astrocytes regulate cortical state switching in vivo. Proc Natl Acad Sci USA 2016 ; 113 : ع2675-84.

20. Sweeney P, Qi Y, Xu Z, Yang Y. Activation of hypothalamic astrocytes suppresses feeding without altering emotional states. Glia $2016 ; 64: 2263-73$.

21. Roth BL. DREADDs for Neuroscientists. Neuron 2016 ; 89 : 683-94.

22. Adamsky A, Kol A, Kreisel T, et al. Astrocytic activation generates de novo neuronal potentiation and memory enhancement. Cell $2018 ; 174$ : 59-71 el4.

23. Martin-Fernandez M, Jamison S, Robin LM, et al. Synapse-specific astrocyte gating of amygdala-related behavior. Nat Neurosci 2017; $20: 1540-8$.

24. Bull C, Freitas KC, Zou S, et al. Rat nucleus accumbens core astrocytes modulate reward and the motivation to self-administer ethanol after abstinence. Neuropsychopharmacology 2014 ; 39 : 2835-45.

25. Zhang $Y$, Chen K, Sloan SA, et al. An RNA-sequencing transcriptome and splicing database of glia, neurons, and vascular cells of the cerebral cortex. J Neurosci 2014 ; 34 : 11929-47.

26. Hedlund $\varepsilon$, Deng $\varrho$. Single-cell RNA sequencing: technical advancements and biological applications. Mol Aspects Med 2018 ; 59 : 36-46.

27. Sala Frigerio C, Wolfs L, Fattorelli N, et al. The major risk factors for alzheimer's disease: age, sex, and genes modulate the microglia response to abeta plaques. Cell Rep 2019; 27 : 1293-306 e6.

28. Levy $\varepsilon$, Slavov N. Single cell protein analysis for systems biology. Essays Biochem $2018 ; 62$ : 595-605.

29. Sharma K, Schmitt S, Bergner CG, et al. Cell type- and brain region-resolved mouse brain proteome. Nat Neurosci 2015 ; 18 : 1819-31.

30. Boza-Serrano A, Yang Y, Paulus A, Deierborg T. Innate immune alterations are elicited in microglial cells before plaque deposition in the Alzheimer's disease mouse model 5xFAD. Sci Rep 2018; $8: 1550$.

31. Brodin P. The biology of the cell - insights from mass cytometry. F\&BS $2019 ; 286: 1514-22$.

32. Bottcher C, Schlickeiser S, Sneeboer MAM, et al. Human microglia regional heterogeneity and phenotypes determined by multiplexed single-cell mass cytometry. Nat Neurosci $2019 ; 22: 78-90$

33. Strell C, Hilscher MM, Laxman N, et al. Placing RNA in context and space methods for spatially resolved transcriptomics. FEBS J 2019; 286 : 1468-81.

34. Poulopoulos A, Murphy AJ, Ozkan A, et al. Subcellular transcriptomes and proteomes of developing axon projections in the cerebral cortex. Nature $2019 ; 565: 356-60$.

35. Moller T, Boddeke HW. Glial cells as drug targets: What does it take? Glia $2016 ; 64: 1742-54$.

36. Heath F, Hurley SA, Johansen-Berg H, Sampaio-Baptista C. Advances in noninvasive myelin imaging. Dev Neurobiol 2018 ; 78: 136-51.

37. Aiello M, Cavaliere C, Fiorenza D, et al. Neuroinflammation in neurodegenerative diseases: current multi-modal imaging studies and future opportunities for hybrid PET/MRI. Neuroscience 2018 ; 403 : 125-35. 


\section{RéFÉRENCES}

38. Barros LF, Bolanos JP, Bonvento G, et al. Current technical approaches to brain energy metabolism. Glia $2018 ; 66: 1138-59$.

39. Valori CF, Guidotti G, Brambilla L, Rossi D. Astrocytes: emerging therapeutic targets in neurological disorders. Trends Mol Med $2019 ; 25: 750-9$.

40. Pian $\mathrm{H}$, Kang X, Hu J, et al. Reversing a model of Parkinson's disease with in situ converted nigral neurons. Nature $2020 ; 582: 550-6$.

41. Zhou H, Su J, Hu X, et al. Glia-to-neuron conversion by crispr-casrx alleviates symptoms of neurological disease in mice. Cell 2020 ; $181: 590-603$ el6.

42. Elsayed M, Magistretti PJ. A New outlook on mental illnesses: glial involvement beyond the glue. Front Cell neurosci $2015 ; 9: 468$.
43. Agid Y, Fan X. L'autre moitié du cerveau, les cellules gliales. Med Sci (Paris) $2019 ; 35: 199-200$.

44. Agid Y, Magistretti P. L'homme glial. Paris : Odile Jacob, 2018.

45. Zalc B, Rosier F. La myéline : le turbo du cerveau. Paris : Odile Jacob, 2016.

46. Jordan B. CRISPR : le Nobel, enfin... Med Sci (Paris) 2021 ; $37: 77-80$
TIRÉS À PART

H. Hirbec

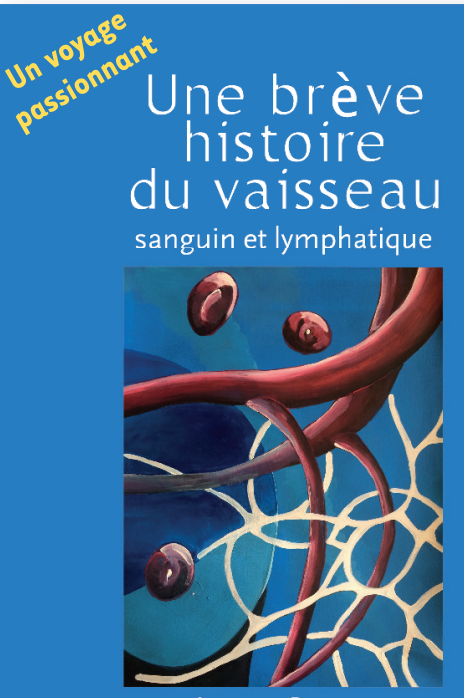

ANDREAS BIKFALYI e livre, intéressant et lisible à la fois pour le spécialiste et le grand public, apporte des observations originales et nouvelles concernant l'angiogenèse, et notamment l'histoire des différentes découvertes, et discute les aspects et les concepts plus généraux en les plaçant dans le contexte de la philosophie des sciences.

Facile à lire, bien illustré, cet ouvrage cherche à comprendre et à faire comprendre les enjeux de la recherche sur l'arbre vasculaire en développement et en pathologie. II intéressera non seulement les étudiants et post-doctorants en biologie, mais aussi les chercheurs actifs dans ce domaine de recherche ainsi que toute personne intéressée par la biologie et la médecine et par l'histoire des sciences. Un voyage passionnant à travers l'histoire et les concepts les plus actuels concernant les recherches sur le vaisseau sanguin.

Andreas Bikfalvi est Professeur à l'université de Bordeaux et Directeur d'une unité de recherche Inserm sur le cancer et la biologie vasculaire. Il est, par ailleurs, membre senior de l'Institut Universitaire de France (IUF) et reconnu internationalement pour ses recherches dans le domaine de l'angiogenèse tumorale.

\section{À retourner à EDP Sciences, 17, avenue du Hoggar, 91944 Les Ulis Cedex, France Tél. : 0149856069 - Fax : 0149850345 - ع-mail : françois.flori@edpsciences.org}

NOM :

Prénom :

Adresse :

Code postal :

Ville :

Pays :

Fonction :

Je souhaite recevoir

Une brève histoire de vaisseau : $25 €+3 €$ de port $=28 € \pi C$

$\square$ Par chèque, à l'ordre de EDP Sciences

$\square$ Par carte bancaire : $\quad \square$ Visa $\square$ Eurocard/Mastercard

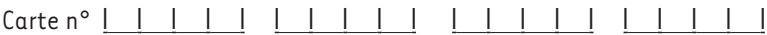

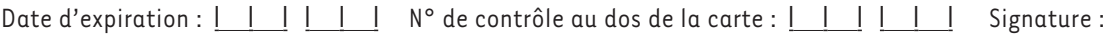

